\title{
Parainfluenza: A Respiratory System Illness
}

\author{
Aakansha Tiwari, Seema Agarwal", Garima Pandey \\ College of Veterinary and Animal Sciences, Govind Ballabh Pant University of Agriculture and Technology, Uttarakhand, India
}

\begin{abstract}
Parainfluenza is a highly contagious disease of animals which affects mainly the respiratory system of the body. The disease is caused by the Parainfluenza viruses which are the enveloped viruses belonging to the Paramyxoviridae family under order Mononegavirales. These viruses affect the respiratory system of bovine, canine and murine species. The virus is transmitted through direct contact with infected animals or by aerosols produced through exhalation, coughing or sneezing. After inhalation, the virus invades the respiratory epithelium and affects the alveolar macrophage which increases susceptibility to secondary infection. The infection is transmitted rapidly among stressed animal. It is manifested by an increase in body temperature, lacrimation, and serous discharge from the nasal opening, coughing, depression, and dyspnoea. Diagnosis of this viral infection can be done on the basis of various serological assays, immunohistochemistry, RT-PCR methods. Treatment against it is mainly symptomatic and directed primarily towards combating the secondary bacterial infections. Vaccination is the best prevention strategy against the disease occurrence and various combined vaccines are available in the market.
\end{abstract}

Keywords:

Parainfluenza $\bullet$ Vaccination $\bullet$ Paramyxoviridae $\cdot$ Infection

\section{Introduction}

Viruses and bacteria have always been the major contributors of the various infections of respiratory tract in the animals worldwide and in this, viruses are the ones to initiate the infection [1]. The main viruses affecting the respiratory system of animals include: BVDV, BHV-1, BRSV, PIV-3 and BAV. Among these, the Parainfluenza group of viruses are extremely infectious and a major cause of acute infection of respiratory system in animals. These are non-segmented, enveloped, - ve sense ss-RNA viruses. Under Paramyxoviridae family, the genus Respirovirus incorporates human parainfluenza viruses 1 and 3, Bovine parainfluenza virus 3 (BPIV 3) and Sendai viruses. Human parainfluenza viruses 2 and 4 and Canine parainfluenza virus 5 (CPIV 5) comes under the genus Rubulavirus. Species designations are the method which is most often used to distinguish the different parainfluenza viruses. All these viruses are less limited to host species boundaries. These parainfluenza viruses were first discovered in the era of late 1950s. The three most important parainfluenza diseases in animals include: Bovine parainfluenza, Canine parainfluenza and Murine parainfluenza.

\section{Etiology}

In case of bovines, Bovine parainfluenza 3 is responsible for causing Parainfluenza (it shows genetic similarity to human parainfluenza virus. The isolation of this virus (USA) was for the first time done from the nasal discharge of calves showing clinical signs of shipping fever, including rapid onset of fever, nasal discharge, coughing and other respiratory signs, lacrimation, conjunctivitis and lack of appetite. Canine parainfluenza is caused by Canine parainfluenza virus (Simian parainfluenza virus 5). Simian virus 5 was primarily isolated from monkey cell cultures, but it is now it has been deduced that dogs are the natural host to this virus. Murine parainfluenza is caused by Murine parainfluenza virus 1 (MPIV 1 ), which is also considered as Sendai virus. Sendai virus was discovered in 1952, after inoculation of lung material from pneumonic human infants into laboratory mice in the course of attempts to isolate human respiratory viruses. All these viruses belong to the Family Paramyxoviridae.

\section{Host Range}

BPIV 3 has a worldwide distribution and can infect many ungulate species including cattle, sheep, goats, and wild ruminants, also humans and nonhuman primates. CPIV3 causes infection in dogs and with this virus it is still under debate that whether it is a cause of zoonotic infection or not. Sendai virus (MPIV) infection occurs worldwide in laboratory and wild rodents.

\section{Transmission}

The important routes of transmission of the virus in susceptible animals are aerosol and fomites contaminated with nasal discharges, as the virus is exclusively a respiratory tract pathogen [2]. Virus shedding starts 6-8 days after infection. Mode of transmission determines the tropism and magnitude of primary infection, which in turn influences the tropism and magnitude of reinfection [3].

\section{Pathogenesis and Pathology}

Epithelial cells of the respiratory tract are the primary target cells for BPIV3, but type II pneumocytes and alveolar macrophages are also infected, sometimes with the presence of acidophilic intracytoplasmic and/or

Correspondence to: Seema Agarwal, College of Veterinary and Animal Sciences, Govind Ballabh Pant University of Agriculture and Technology, Uttarakhand, India, Tel: +923217415605, +91 9756753533; E-mail: seema_patho@rediffmail.com,dr.seemapatho@gmail.com

Received: March 14, 2020; Accepted: June 23, 2020; Published: June 30, 2020

Copyright: (c) 2020 Tiwari A. This is an open-access article distributed under the terms of the Creative Commons Attribution License, which permits unrestricted use, distribution, and reproduction in any medium, provided the original author and source are credited. 
intranuclear inclusion bodies. Infection results in the necrosis. Inflammation of small airways in the lung's specifically bronchiolitis and bronchitis along with the accumulation of cellular exudate in the lumen of affected airways. Upon intranasal or intratracheal inoculation of BPIV3 alone, calves show only mild fever and serous nasal discharge.

The CPIV causes destruction of the ciliated epithelial cells of the respiratory tract. This virus causes in apparent infection or mild respiratory disease in dogs and the virus has been incriminated as an uncommon cause of congenital hydrocephalus also. CPIV5 is implicated in the pathogenesis of the acute respiratory disease of canines (kennel cough syndrome), and more seriously, chronic respiratory disease may develop when additional microbial or viral agents, poor hygiene, or stress complicate the infections.

MPIV is largely non-cytolytic and selectively infects respiratory epithelium of nose, trachea, and bronchioles, as well as type II pneumocytes. An endopeptidase similar to clotting factor $\mathrm{Xa}$ that is secreted by Clara cells within the bronchiolar epithelium of rats and mice is responsible for cleavage of the $\mathrm{F}$ protein, thereby allowing the virus to replicate and amplify within the respiratory tract. Disease is characterized by necrotizing rhinitis, tracheitis, bronchiolitis, and interstitial pneumonia arises during the "immune" phase of infection, wherein cytotoxic $T$ cells destroy virus-infected cells (clinical disease occurs in fully immunocompetent mice). Animals devoid of cellular immunity, such as nude mice, do not develop the pathognomonic immunemediated necrotizing bronchiolitis; rather develop a chronic progressive interstitial pneumonia. Laboratory rats, other rodents, and lagomorphs usually develop very mild or subclinical infections.

\section{Clinical Signs and Symptoms}

In calves, lambs, and goat kids, infection is generally subclinical, but sometimes may be manifest as fever, lacrimation, serous nasal discharge, depression, dyspnea, and coughing [4]. Some animals may develop broncho-interstitial pneumonia that selectively affects the antero-ventral portions of the lungs. Sometimes there may be a predisposition to the bacterial infection by Mannheimiahaemolytica, characterized by purulent nasal discharge, cough, rapid respiration, anorexia, fever, general malaise, and substantial mortality from acute fibrinous bronchopneumonia [5].

Coughing is the most common sign of illness in canines. In severe cases (mostly in malnourished or young dogs) there is also conjunctivitis, tonsillitis, anorexia, and lethargy.

Affected mice exhibit a roughened hair coat, crusting of the eyes, dyspnea, and mortality in adult and post-weaning aged mice, weight loss, and fetal resorption in pregnant animals. Athymic nude and severe combined immunodeficient mice develop chronic wasting disease with progressive weight loss and dyspnea. Immunocompetent mice that survive clinical infection recover with no persistence of the virus. Infection of other laboratory rodents and rabbits is generally subclinical or mild.

\section{Diagnosis}

Primarily virus isolation and serology demonstrate increasing antibody titres. The virus can be isolated in numerous cell culture systems (monkey kidney, Vero, and BHK-21 cells with trypsin in the culture medium) and embryonated eggs. The presence of virus is confirmed by immunofluorescence or immunohistochemical staining of infected monolayers. Serological assays include hemagglutination-inhibition and virus neutralization. Nasal swabs or tracheal wash fluids are the samples of choice for virus detection and virus can also be recovered from the nasal discharges. The virus can be identified in nasal discharges or respiratory tissues by immunofluorescence staining, reverse transcriptase-polymerase chain reaction tests, or immunohistochemistry. RT-PCR is gold standard for rapid testing and confirmation of isolates [6].

\section{Prevention and Control}

Once the infection is diagnosed, depopulation, disinfection of premises and screening of newly introduced animals in the herd is required for controlling further infection. In this view all-in all-out system can be practiced. Overcrowding of herds, humidity and stress to the animals should be avoided. Inactivated and live-attenuated virus vaccines through intranasal and parenteral route of administration are available that induce protective antibodies. Typically, combination of vaccines have been formulated that include various combinations of protective antigens of bovine herpesvirus 1 (Infectious bovine rhinotracheitis virus), bovine viral diarrhoea virus, bovine adeno virus, bovine respiratory syncytial virus, and Mannheimiahaemolytica. There are various combination of vaccinesavailable against various respiratory pathogens being marketed by different trade names such as: Nobivac Canine 1-DAPPV, Nobivac Canine 1-DAPPV+CV, Nobivac Canine 1-DAPPVL2, Nobivac Canine 1-DAPPVL2+CV, Nobivac Canine 1-DAPPV +L4, Nobivac Intra-Trac KC, Nobivac Intra-Trac3, Nobivac Intra-Trac3 ADT.

\section{References}

1. Valarcher, JF, Hägglund, S. "Viral respiratory infections in cattle, Proceedings of the 24th World Buiatrics Congress", Nice, France. (2006).

2. Goldmann, DA. "Transmission of viral respiratory infections in the home". Pediatr Infect Dis J19(2000): S97-102.

3. Burke, CW, Bridges, O, Brown S, and Rahija R, et al. "Mode of Parainfluenza Virus Transmission Determines the Dynamics of Primary Infection and Protection from Reinfection". PLoSPathog 9(2013): e1003786.

4. Bryson, DG. "Parainfluenza-3 Virus in Cattle In Virus infections in Ruminants". Edited by Dinter Z, Morein, B. Amsterdam: Elsevier publishers (1990): 319-333.

5. Haanes, EJ, Guimond, P, and Wardley, R. "The bovine parainfluenza virus type-3 (BPIV-3) hemagglutinin/neuraminidase glycoprotein expressed in baculovirus protects calves against experimental BPIV-3 challenge". Vaccine 15(1997): 730-738.

6. Corne, JM, Green, S, Sanderson, G, and Caul, EO, et al. "A multiplex RTPCR for the detection of parainfluenza viruses 1-3 in clinical samples". $J$ Virol Methods 82(1999): 9-18.

How to cite this article: Tiwari A, Agarwal S, Pandey G. "Parainfluenza: A Respiratory System Illness". J Infect Dis Med 5 (2020) doi: 10.37421/jidm. 2020.5.142 